\title{
Weak ferromagnetism in Mn nanochains on the CuN surface
}

\author{
A.N. Rudenko ${ }^{1,2}$, V.V. Mazurenko ${ }^{1,2}$, V.I. Anisimov ${ }^{1,3}$ and A.I. Lichtenstein ${ }^{2}$ \\ ${ }^{1}$ Theoretical Physics and Applied Mathematics Department, \\ Urals State Technical University, Mira Street 19, 620002 Ekaterinburg, Russia \\ ${ }^{2}$ Institute of Theoretical Physics, University of Hamburg, Jungiusstrasse 9, 20355 Hamburg, Germany \\ ${ }^{3}$ Institute of Metal Physics, Russian Academy of Sciences, 620219 Ekaterinburg GSP-170, Russia
}

(Dated: August 11, 2021)

\begin{abstract}
We investigate electronic and magnetic structures of the Mn chains supported on the CuN surface using first-principle LSDA and LDA+U calculations. The isotropic exchange integrals and anisotropic Dzyaloshinskii-Moriya interactions between Mn atoms are calculated using Green function formalism. It is shown that the account of lattice relaxation and on-site Coulomb interaction are important for accurate description of magnetic properties of the investigated nanosystems. We predict a weak ferromagnetism phenomenon in the $\mathrm{Mn}$ antiferromagnetic nanochains on the $\mathrm{CuN}$ surface. The value of a net magnetic moment and direction of spin canting are calculated. We show that some experimental features may be explained using anisotropic exchange interactions.
\end{abstract}

PACS numbers: 73.22.-f, 75.30.Et

\section{INTRODUCTION}

The investigation of magnetic nanomaterials is important part of nanoscience and exerts influence on progress in different sectors of technology (such as medicine devices for therapy and diagnostics,$\frac{1,2}{,}$ magnetic data storage systems, $\frac{3}{3}$ etc). In the presence of intrinsic magnetic moment scientists can change a physical properties of nanomaterials by applying external magnetic field. The technological applications of magnetic nanomaterials should base on an accurate control of the coupling between individual spins.

Recently, Hirjibehedin et al $\underline{\underline{4}}$ have reported the fabrication of Mn nanoparticles in the form of linear chains that display truly collective quantum behaviour. Using local spin-excitation spectroscopy technique, based on inelastic scanning tunneling microscopy (STM), they were able to show how the quantum properties of this system depend on the number of atoms involved. They demonstrated an innovative method to measure and control these magnetic interactions. The experimental spectrum was analyzed using the simplest form of Heisenberg model with exchange interaction only between nearest neighbors. However, there are a number of experimental results which cannot be explained by authors of Ref.4 using the Heisenberg model: (i) zero-field splitting which grows in energy with increasing chain length, (ii) the different zero-field energy of the $m= \pm 1$ and $m=0$ excited states and (iii) asymmetry of the spectra with respect to voltage polarity.

Jones and $\operatorname{Lin}^{5}$ have applied GGA $+\mathrm{U}$ approach to describe the electronic and magnetic structures of single and pair of $\mathrm{Mn}$ atoms on the $\mathrm{CuN}(100)$ surface. The performed spin-density analysis shows that $\mathrm{Mn}$ atoms on such surface preserve their atomic spins $S=\frac{5}{2}$. This result agrees with STM measurement. $\underline{\underline{4}}$ Electron-density change and surface relaxation due to $\mathrm{Mn}$ atoms are also analyzed in Ref:5.

The combination of experimental STM approach and theoretical ab-initio methods $\underline{\underline{\underline{6}}}$ has been used in order to describe the large magnetic anisotropy of individual $\mathrm{Mn}$ and $\mathrm{Fe}$ atoms on the $\mathrm{CuN}$ surface. The authors of the paper ${ }^{6}$ have provided the detailed phenomenological picture of magnetic anisotropy and concluded that in case of manganese system the easy axis is oriented out-of-plane.

In this paper we show that the local distortion of the system results in a superexchange interaction between $\mathrm{Mn}$ atoms through $\mathrm{N}$ atoms. Isotropic exchange interactions are calculated using Green functions approach and total energies difference method. Using full diagonalization of Heisenberg Hamiltonian with calculated isotropic exchange integrals we estimate the energies of first magnetic excitations. The results are in good agreement with experimental data.

In the previous theoretical investigations a noncollinear magnetic ground state for nanostructures on non-magnetic surfaces ${ }^{7,8}$ and magnetic $\frac{9}{}$ were proposed. For instance, the results for the non-collinear triangular compact trimer of $\mathrm{Cr}$ on the $\mathrm{Au}(111)^{7}$ predict that the angle between each pair of moments equals to $120^{\circ}$ and the total spin moment is zero. Therefore, the noncollinearity is result of frustration of magnetic interactions.

In the paper $\stackrel{8}{\underline{8}}$ authors have investigated different geometries of $\mathrm{Fe}, \mathrm{Mn}$ and $\mathrm{Cr}$ atoms on the $\mathrm{Cu}(111)$ surface. The Fe clusters were found to be ferromagnetically ordered. Whereas for the $\mathrm{Mn}$ and Cr clusters an antiferromagnetic exchange interactions between nearest neighbours have been found. The antiferromagnetic couplings produce either collinear or non-collinear magnetic structures due to frustration of cluster geometry.

An interesting results for the trimer and tetramer configurations of $\mathrm{Mn}$ and $\mathrm{Cr}$ atoms on the magnetic $\mathrm{Ni}(111)$ surface were obtained in the paper $\stackrel{\underline{9}}{=}$ One should stressed that there are two types of a magnetic frustration: (i) frustration within adcluster and (ii) frustration arising from competing magnetic interactions between the adclusters and the surface atoms. 
Thus, one can conclude that the only known source for spin non-collinearity of magnetic clusters on nonmagnetic $3 \mathrm{~d}$ surface is geometrical frustration which results in magnetic frustration. In this paper we propose a new source of spin non-collinearity for nanosystems on a surface. According to our calculations a local distortion between $\mathrm{Mn}$ atoms in the nanochain results in the strong Dzyaloshinskii-Moriya (DM) interaction. An important role plays the displacement of $\mathrm{N}$ atom from the surface. Based on first-principle calculations of the Dzyaloshinskii-Moriya interactions between magnetic moments we point out that the Mn nanochains on the $\mathrm{CuN}$ demonstrate a weak ferromagnetism phenomenon. We have estimated the value of a net magnetic moment and direction of spin canting. These results are also confirmed by direct $\mathrm{LDA}+\mathrm{U}+\mathrm{SO}$ calculations.

The paper is organized as follows. In Section II we describe the methods of the investigation. In Section III $\mathrm{A}$ and III B we present the results of LSDA and LDA+U calculations, respectively. The analysis and comparison of obtained exchange interactions with experimental data are presented in Section III C. Section IV is devoted to the analysis of zero-field energy splitting observed in STM experiment and in section $\mathrm{V}$ we briefly summarize our results.

\section{METHODS OF INVESTIGATION}

\section{A. DFT calculation details}

We have used two complementary approaches for investigations of an electronic and magnetic properties of $\mathrm{Mn}$ nanochains on the $\mathrm{CuN}$ surface.

(i) First-principles total-energy and force calculations were carried out using the projected augmented-wave (PAW) method ${ }^{10}$ as implemented in the Vienna ab initio simulation package (VASP) $, 11,12$ Exchange and correlation effects have been taken into account using LSDA and $\mathrm{LDA}+\mathrm{U}^{13}$ approaches. In all cases under investigation we used an energy cutoff of $400 \mathrm{eV}$ in the plane-wave basis construction and the energy convergence criteria of $10^{-4} \mathrm{eV}$. The atomic positions of considered systems were relaxed with residual forces less than $0.01 \mathrm{eV} / \AA$. For the Brillouin zone integration, a $(4 \times 4 \times 1)$ Monkhorst-Pack mesh $^{14}$ and Gaussian-smearing approach with $\sigma=0.2 \mathrm{eV}$ were used.

To simulate structure of the unit cell we have used a supercell approach. Structure of the supercell has consisted of two-layer $(2 \times(\mathrm{n}+1)) \mathrm{Cu}(100)$ surface, $\mathrm{N}$ atoms embedded into upper $\mathrm{Cu}$-layer, $\mathrm{Mn}_{n}$-chains placed on the top of the $\mathrm{CuN}$-surface and vacuum region of $10 \AA$. Lattice constant for $\mathrm{Cu}$ was chosen to be $3.63 \AA$, which gives a minimal value of the total energy in calculation of the bulk fcc $\mathrm{Cu}$. Lower layer of $\mathrm{Cu}$ has been fixed under relaxation.

(ii) We have also used the Tight Binding Linear-MufinTin-Orbital Atomic Sphere Approximation (LMTO) $\operatorname{method}^{15}$ in terms of the conventional local density approximation taking into account the on-site Coulomb interaction $\mathrm{LDA}+\mathrm{U}$ and spin-orbit coupling

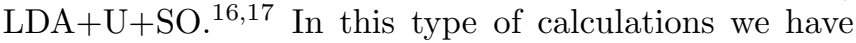
used the relaxed structures obtained by PAW approach. The radii of atomic spheres were $\mathrm{r}(\mathrm{Mn})=1.137 \AA$, $\mathrm{r}(\mathrm{Cu})=1.322 \AA$ and $\mathrm{r}(\mathrm{N})=0.793 \AA$. In order to fill the empty space of the unit cell required number of empty spheres were added.

\section{B. Spin Hamiltonian approach}

The main aim of our investigation is first-principle determination of parameters of the following spin Hamiltonian:

$$
H=H_{H e i s}+H_{D M}
$$

where Heisenberg ${ }^{18}$ energy term is

$$
H_{H e i s}=\sum_{i<j} J_{i j} \vec{S}_{i} \vec{S}_{j},
$$

and Dzyaloshinskii-Moriya ${ }^{19}$ energy term is

$$
H_{D M}=\sum_{i<j} \vec{D}_{i j}\left[\vec{S}_{i} \times \vec{S}_{j}\right]
$$

In order to calculate the isotropic exchange interactions $\mathrm{J}_{i j}$, in Eq.(2) between magnetic moments of Mn atoms we have used two different approaches. (i) The total energy difference method on the basis of PAW results. The main idea of this method is that the isotropic exchange interaction defines through the energy differences between different magnetic configurations. For instance, in case of Mn-dimer, spin Hamiltonian of the system can be written in the following form:

$$
H=J \vec{S}_{1} \cdot \vec{S}_{2}
$$

The corresponding total energies of the ferromagnetic and antiferromagnetic configurations of two classical spins are given by

$$
E_{F M}=J S^{2}
$$

and

$$
E_{A F M}=-J S^{2}
$$

Therefore, the exchange interaction $J$ is expressed in the following form:

$$
J=\frac{E_{F M}-E_{A F M}}{2 S^{2}} .
$$

(ii) From the other hand, based on LMTO results one can calculate the isotropic exchange integrals and Dzyaloshinskii-Moriya interactions between magnetic 
moments of $\mathrm{Mn}$ atoms $\left(\mathrm{S}=\frac{5}{2}\right)$ using the local force theorem and Green functions formalism $20,21,22$

$$
\begin{gathered}
J_{i j}=\frac{1}{2 \pi S^{2}} \int_{-\infty}^{E_{F}} d \epsilon \operatorname{Im} \\
\sum_{\substack{m, m^{\prime} \\
m^{\prime \prime}, m^{\prime \prime \prime}}}\left(\Delta_{i}^{m m^{\prime}} G_{i j \downarrow}^{m^{\prime} m^{\prime \prime}} \Delta_{j}^{m^{\prime \prime} m^{\prime \prime \prime}} G_{j i \uparrow}^{m^{\prime \prime \prime} m}\right),
\end{gathered}
$$

where $m\left(m^{\prime}, m^{\prime \prime}, m^{\prime \prime \prime}\right)$ is magnetic quantum number and the on-site potential $\Delta_{i}^{m m^{\prime}}=H_{i i \uparrow}^{m m^{\prime}}-H_{i i \downarrow}^{m m^{\prime}}$. The Green function is calculated in the following way:

$$
G_{i j \sigma}^{m m^{\prime}}(\epsilon)=\sum_{\mathbf{k}, n} \frac{c_{i \sigma}^{m n}(\mathbf{k}) c_{j \sigma}^{m^{\prime} n *}(\mathbf{k})}{\epsilon-E_{\sigma}^{n}(\mathbf{k})} .
$$

Here $c_{i \sigma}^{m n}$ is a component of the $n$-th eigenstate, $\mathrm{E}_{\sigma}^{n}$ is the corresponding eigenvalue and $\mathbf{k}$ is quasimomentum in the first Brillouin Zone.

In turn the Dzyaloshinskii-Moriya interaction, Eq.(3) can be calculated through the account of spin-orbit coupling in the second variation of total energy of the system over the small deviations of magnetic moments from the collinear ground state: ${ }^{21,23}$

$$
\begin{array}{r}
D_{i j}^{z}=-\frac{1}{2 \pi S^{2}} \operatorname{Re} \int_{-\infty}^{E_{F}} d \epsilon \sum_{k} \\
\times\left(\Delta_{i} G_{i k}^{\downarrow} H_{k \downarrow \downarrow}^{s o} G_{k j}^{\downarrow} \Delta_{j} G_{j i}^{\uparrow}-\Delta_{i} G_{i k}^{\uparrow} H_{k \uparrow \uparrow}^{s o} G_{k j}^{\uparrow} \Delta_{j} G_{j i}^{\downarrow}\right. \\
\left.+\Delta_{i} G_{i j}^{\downarrow} \Delta_{j} G_{j k}^{\uparrow} H_{k \uparrow \uparrow}^{s o} G_{k i}^{\uparrow}-\Delta_{i} G_{i j}^{\uparrow} \Delta_{j} G_{j k}^{\downarrow} H_{k \downarrow \downarrow}^{s o} G_{k i}^{\downarrow}\right),
\end{array}
$$

where $H_{k}^{s o}=\lambda_{k} \vec{L} \vec{S}$ and $\lambda_{k}$ is spin-orbit coupling constant for site $k$. Here we present only $z$ component of Dzyaloshinskii-Moriya vector. $x$ and $y$ components can be obtained from the $z$ ones by rotation of the coordinate system.

\section{RESULTS}

\section{A. LSDA results}

We have performed LSDA calculation of antiferromagnetic Mn-dimer supported on the CuN surface. Fig:1 shows the relaxed structure of the Mn-dimer obtained within LSDA method using PAW approach. The information about structure of relaxed system is presented in Table I. One can see that Mn-N-Mn bond angle of $171^{\circ}$ is close to $180^{\circ}$ and corresponds to the maximum of superexchange interaction between $3 \mathrm{~d}$ atoms.

The calculated total and partial densities of states obtained using PAW approach are presented in Fig. 2. The valence band contains low and high energy parts which are separated by $6 \mathrm{eV}$. The low energy states located around $-15 \mathrm{eV}$ are mainly $\mathrm{N}$ ones. In the region from -7 $\mathrm{eV}$ to $5 \mathrm{eV}$ all states are highly mixed.

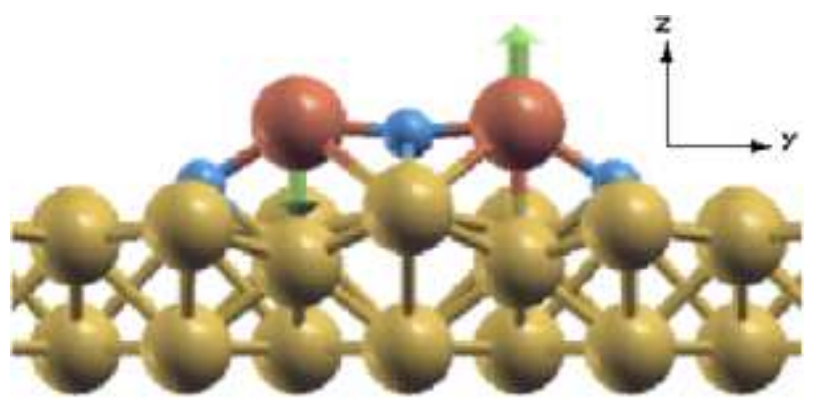

FIG. 1: The projection on yz-plane of relaxed structure of Mn-dimer supported on the $\mathrm{CuN}$ obtained using LSDA approach. Yellow, blue and red spheres correspond to $\mathrm{Cu}$, $\mathrm{N}$ and $\mathrm{Mn}$ atoms, respectively. Green arrows correspond to direction of atomic magnetic moments.

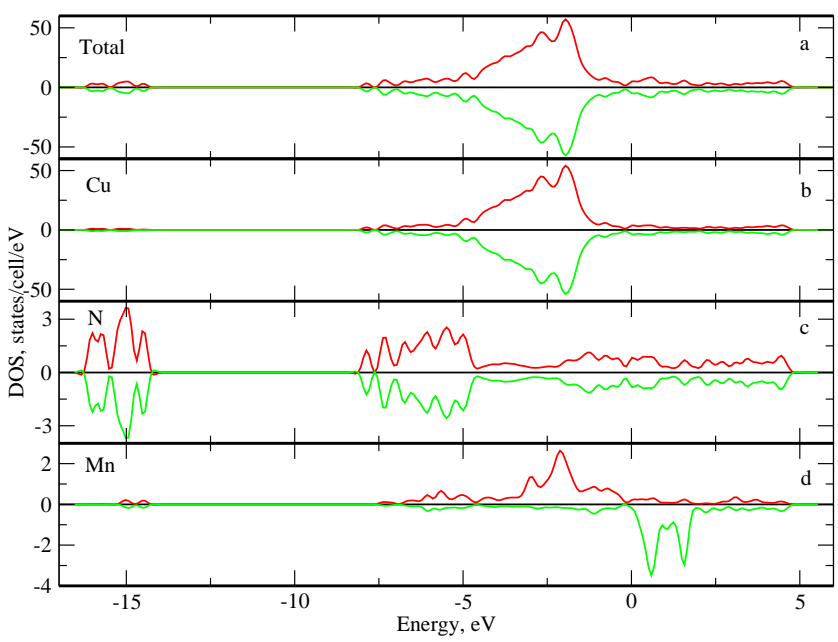

FIG. 2: Total and partial density of states obtained using LSDA calculations. (a) Total DOS, (b) Projected DOS onto $\mathrm{Cu}$ atoms, (c) Projected DOS onto N atoms, (d) Projected DOS onto Mn atom. Fermi level corresponds to $0 \mathrm{eV}$.

The calculated values of magnetic moments of $\mathrm{Mn}$ atoms within LMTO and PAW approaches are $3.70 \mu_{B}$ and $3.35 \mu_{B}$, respectively. These values are smaller than experimentally observed spin $\frac{5}{2}$. Moreover, the calculated exchange parameter $J_{i j}$ within Green functions approach, Eq.(8) is $20.4 \mathrm{meV}$, whereas total energies difference method value, Eq.(7) is $24.8 \mathrm{meV}$. These values at least three times larger than that experimentally observed. Thus, one can see that the main electronic and magnetic properties of the investigated nanosystem cannot be correctly reproduced within the LSDA approach.

\section{B. LDA+U results}

The results of the previous section have demonstrated drawbacks of the LSDA approach to describe the mag- 

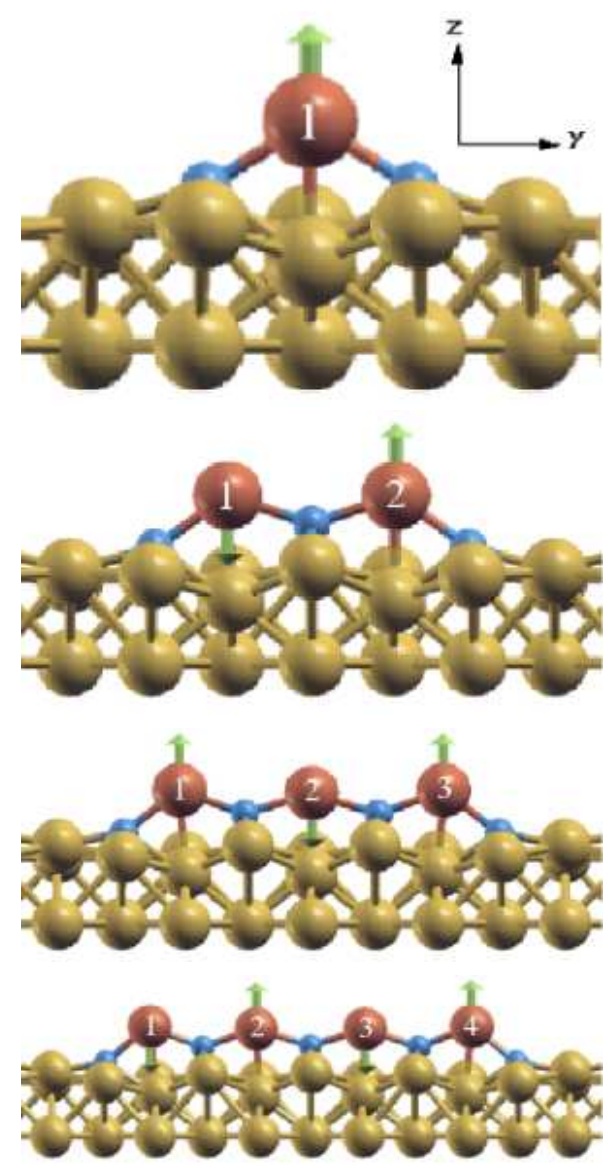

FIG. 3: The yz projections of relaxed structures of Mn chains of $\mathrm{CuN}$ surface for $\mathrm{n}=1 \div 4$. Yellow, blue and red spheres correspond to $\mathrm{Cu}, \mathrm{N}$ and $\mathrm{Mn}$ atoms, respectively. Green arrows correspond to direction of atomic magnetic moments of manganese.

netic properties of the $\mathrm{Mn}$ dimer on the $\mathrm{CuN}$ surface. It is well known problem of local density approximation in respect to transition metal compounds. To overcome this problem we have used the $\mathrm{LDA}+\mathrm{U}$ approach with on-site Coulomb and on-site Hunds interaction parameters of $U=6.0 \mathrm{eV}$ and $J_{H}=0.9 \mathrm{eV}$, respectively. These values are in good agreement with recent first-principle estimations performed in the work $\underline{\underline{6}}$

The relaxed structures of the Mn-chains of different lengths $(\mathrm{n}=1 \div 4)$ obtained within PAW calculations are shown in Fig, 3 . The structural information is presented in Table I. The obtained structures have some interesting geometrical features. Let us analyze the difference between the clean CuN-substrate and the substrate with $\mathrm{Mn}$ adatoms on the top. Presence of $\mathrm{Mn}$ atoms causes some rearrangement of upper layer atoms of $\mathrm{CuN}$ substrate. Generally, this rearrangement concerns the $\mathrm{N}$ atoms. In contrast to clean-CuN surface, the $\mathrm{N}$ atoms of the system with Mn nanochains are significantly shifted from the first layer plane. This fact agrees with results of recent GGA calculations. $\underline{\underline{5}}$ The calculated angle of $\mathrm{Mn}-\mathrm{N}$ -

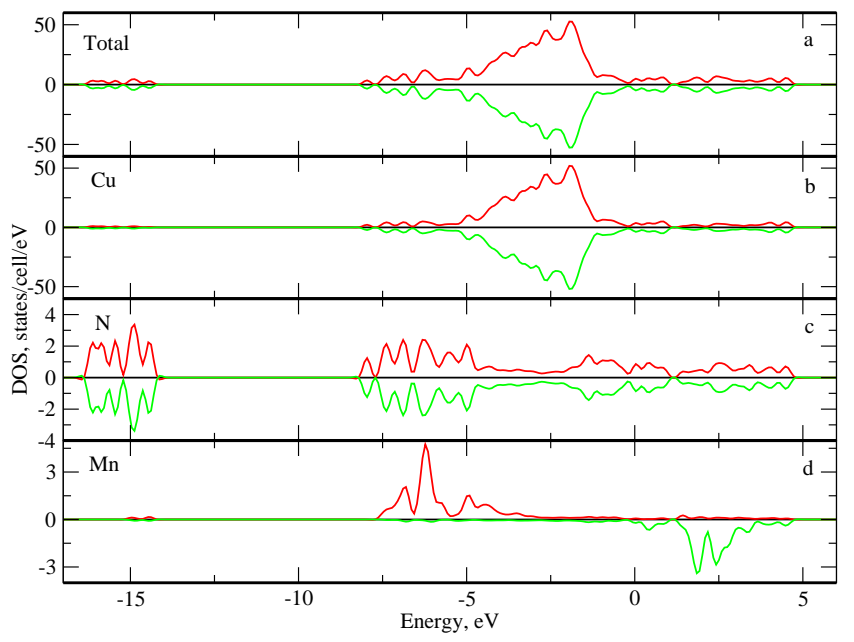

FIG. 4: Total and partial density of states of Mn-dimer on $\mathrm{CuN}$ surface obtained using LDA $+\mathrm{U}$ calculations. (a) Total DOS, (b) Projected DOS onto Cu atoms, (c) Projected DOS onto N atoms, (d) Projected DOS onto Mn atom. Fermi level corresponds to $0 \mathrm{eV}$.

Mn bond within LDA+U approximation equals to $143^{\circ}$. The $\mathrm{N}$ atoms at the edges of chains also have some displacement from the plane in z-direction, but to a smaller extent than $\mathrm{N}$ atoms situated inside the chain.

From a geometrical point of view the important difference between results of LSDA and LDA $+\mathrm{U}$ approaches is the angle of Mn-N-Mn bond. Let us analyze this fact on the level of hopping integral. For simplicity, we assume that there is the only strong hopping between orbitals of two $3 \mathrm{~d}$ atoms. The hopping integral $t_{i j}$ is proportional to $\cos \alpha$, where $\alpha$ is angle of metal-ligand-metal bond. Therefore, within LDA $+\mathrm{U}$ approach the hopping integral is strongly suppressed due to local Coulomb correlations. In turn the isotropic exchange interaction between $\mathrm{Mn}$ atoms in the atomic limit of Hubbard model can be expressed as $J_{i j}=\frac{4 t_{i j}^{2}}{U}$, here $U$ is on-site Coulomb integral. It is clear that on-site Coulomb interaction is another source of suppression of the isotropic exchange interaction in LDA+U in comparison with LSDA approach.

The total and partial densities of states of the dimer and trimer systems obtained using $\mathrm{LDA}+\mathrm{U}$ approximation are presented in Fig 4 and 5, respectively. The calculated value of magnetic moment are listed in Table III. One can see that the values of magnetic moments of Mn atoms within the chains varies from $4.34 \mu_{B}$ (middle atoms) to $4.47 \mu_{B}$ (edge atoms) and now is much closer to experimental values of $S=\frac{5}{2}$. In the case of LMTO results this difference is much smaller.

\section{Isotropic exchange interaction}

The next step of our investigation is determination of Heisenberg exchange interaction parameters in Eq.(2). 


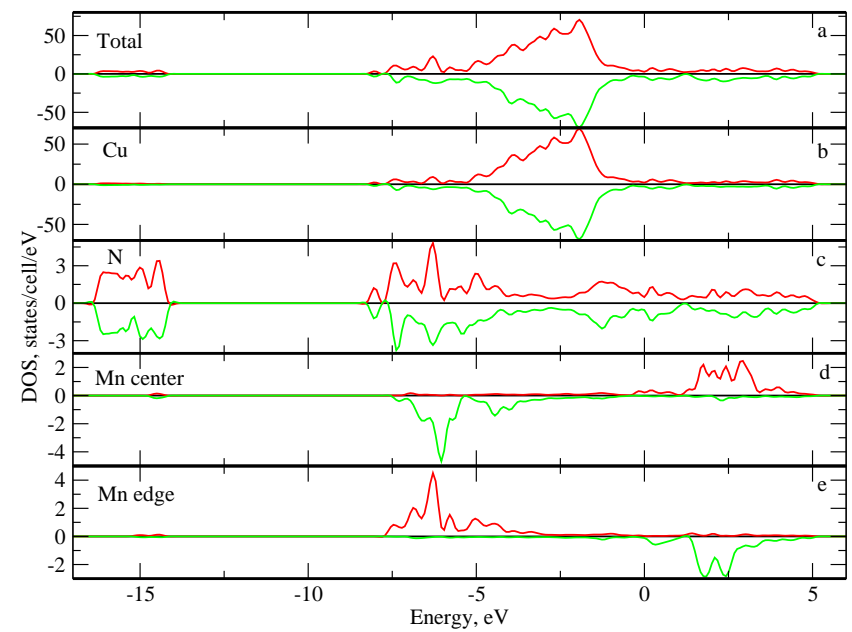

FIG. 5: Total and partial density of states of Mn-trimer chain on $\mathrm{CuN}$ surface obtained using LDA+U calculations. (a) Total DOS, (b) Projected DOS onto $\mathrm{Cu}$ atoms, (c) Projected DOS onto N atoms, (d) Projected DOS onto central Mn atom, (e) Projected DOS onto edge Mn atom.

TABLE I: Structural information about alignment Mn and N atoms on the $\mathrm{Cu}(100)$ surface as result of relaxation in cases of LSDA and LDA+U approaches. The values are distance (in $\AA$ ) between Mn atoms, $z$-coordinates of Mn atoms and N atoms at the center and edge of chain, respectively. $\alpha$ is angle of Mn-N-Mn bond. Zero level of z coordinate corresponds to the lower layer of $\mathrm{Cu}$ atoms. All values are given in $\AA$.

\begin{tabular}{cccccc}
\hline \hline & $d_{M n-M n}$ & $z_{M n}$ & $z_{N}($ center $)$ & $z_{N}($ edge $)$ & $\alpha$ \\
\hline LSDA & 3.58 & 3.66 & 3.49 & 2.66 & $171^{\circ}$ \\
LDA+U & 3.78 & 3.75 & 3.11 & 2.59 & $143^{\circ}$ \\
\hline \hline
\end{tabular}

TABLE II: Values of magnetic moments of Mn atoms (in $\mu_{B}$ ) calculated using LMTO (PAW) method.

\begin{tabular}{lcc}
\hline \hline $\mathrm{n}$ & \multicolumn{1}{c}{$\mathrm{M}_{\text {edge }}$} & \multicolumn{1}{c}{$\mathrm{M}_{\text {center }}$} \\
\hline 1 & - & $4.46(4.48)$ \\
2 & $4.45(4.44)$ & - \\
3 & $4.47(4.47)$ & $4.45(4.34)$ \\
4 & $4.47(4.47)$ & $4.40(4.35)$ \\
\hline \hline
\end{tabular}

The magnetic couplings between Mn atoms calculated using Green functions, Eq.(8) and the total energy difference method, Eq.(7) are presented in Table III. One can see that the calculated values of the dimer interaction are in good agreement with experimental value of $6.4 \mathrm{meV}$. The value of isotropic exchange integral between nearest Mn atoms in trimer is smaller than that in dimer system. There is also small ferromagnetic coupling between edge Mn atoms.

In the case of quatromer system there is the difference between exchange integrals $\mathrm{J}_{12}$ and $\mathrm{J}_{23}$. The coupling at the center of the chain is smaller than the coupling at the edge. There is interesting dependence of nearestneighbour exchange interaction according to the chain length. This tendency probably corresponds to oscillations of exchange interaction parameter depending on chain length. It is important to investigate the mechanism of such strong oscillations (see $\mathrm{Mn}_{3}$ results in Table III) of nearest neighbour exchange interactions in $\mathrm{Mn}_{n^{-}}$ nanochains. Such analysis is left for future investigation.

Using full diagonalization procedure of ALPS library ${ }^{24,25}$ we have calculated the spin excitation spectra of investigated systems. The energies of first excited states are presented in Table[V] Despite of the fact that the $J_{12}$ of trimer has smaller value than in dimer case, weak ferromagnetic $J_{13}$ interaction between edge atoms compensates this difference and gives us opportunity to reproduce experimentally observed excitation energy with reasonable accuracy.

TABLE III: Values of exchange interactions $\mathrm{J}_{i j}$ (in meV) between magnetic moments of Mn atoms calculated using Green function method (TB-LMTO-ASA) method. Values obtained using total energies difference method (PAW) approach are given in parenthesis.

\begin{tabular}{|c|c|c|c|c|}
\hline $\mathrm{J}_{12}$ & $\overline{J_{13}}$ & $\overline{J_{23}}$ & $\overline{J_{34}}$ & $\mathrm{~J}_{24}$ \\
\hline $27.0(6.0)$ & - & - & - & - \\
\hline $34.0(4.2)$ & $-0.09(-0.09)$ & $4.0(4.2)$ & - & $\begin{array}{lll}- & - & -\end{array}$ \\
\hline $45.6(5.2)$ & $-0.07(-0.04)$ & $2.4(4.1)$ & $5.6(5.2)$ & $-0.07(-0.04)$ \\
\hline
\end{tabular}

For quatromer system our results are in excellent agreement with experimental spectrum.

TABLE IV: Energies of first excited states (in meV) of Heisenberg model obtained using ALPS code for the chain systems of different length.

\begin{tabular}{cccc}
\hline \hline$n$ & $E^{\text {exp }}$ & $E_{L M T O}^{\text {calc }}$ & $E_{P A W}^{c a l c}$ \\
\hline 2 & 6.4 & 7.0 & 6.0 \\
3 & 16.0 & 10.5 & 10.9 \\
4 & 2.9 & 3.0 & 2.6 \\
\hline \hline
\end{tabular}

\section{Dzyaloshinskii-Moriya interaction}

From the crystal symmetry point of view there is no inversion center at the point bisecting the straight line between Mn atoms of investigated nanosystems. Therefore, in according with Moriya's rules ${ }^{19}$ a DM coupling exists. First, let us perform a simple geometrical analysis of the symmetry of Dzyaloshinskii-Moriya vector. There are two sources of inversion symmetry breaking in the investigated systems. (i) The first one is the substrate surface. Based on the fact that previous investigations of metallic nanochains on nonmagnetic $3 \mathrm{~d}$ surface has no sign of non-collinearity, one can conclude that surface gives negligible small contribution to anisotropic exchange interaction. (ii) More importantly, the second source of inversion symmetry breaking is vertical displacement of N atom. The Dzyaloshinskii-Moriya vector, $\vec{D}_{12}$ between 
Mn atoms is proportional to $\left[\vec{r} \times \vec{R}_{12}\right], \frac{26}{,}$ where $\vec{R}_{12}$ is a unit vector along the line connecting the magnetic ions and $\vec{r}$ is the shift of the ligand atom from this line (Fig. 6). One can see that in our case $\vec{r}$ and $\vec{R}_{12}$ have $\mathrm{z}$ and y components, respectively. Therefore, the direction of Dzyaloshinskii-Moriya vector is $\mathrm{x}$ axis.

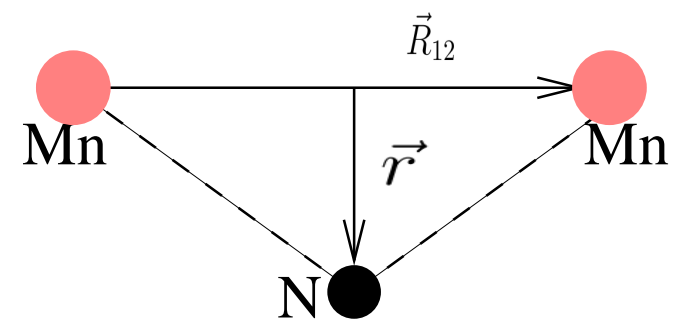

FIG. 6: Schematic representation of displacement of the ligand atom.

The calculated anisotropic exchange interactions, Eq.(10) are presented in Table V] For all systems under consideration the Dzyaloshinskii-Moriya vector lies along $\mathrm{x}$ axis (perpendicular to the Mn chain and parallel to the CuN surface). Therefore, if all spins lie in the yz plane, the canting exists and there is weak ferromagnetism in the system. The ratio between DzyaloshinskiiMoriya and isotropic exchange interactions, $\frac{\left|\vec{D}_{12}\right|}{J_{12}}=0.002$ is the same order of magnitude as in case of well known antiferromagnets $\mathrm{Fe}_{2} \mathrm{O}_{3}$ and $\mathrm{La}_{2} \mathrm{CuO}_{4}$ with weak ferromagnetism.

TABLE V: Values of x component of Dzyaloshinskii-Moriya interactions $\vec{D}_{i j}$ (in meV) between magnetic moments of Mn atoms calculated using Green functions method (Eq.(10)) within $\mathrm{LDA}+\mathrm{U}$ approach.

\begin{tabular}{cccc}
\hline \hline$n$ & $D_{12}^{x}$ & $D_{13}^{x}$ & $D_{23}^{x}$ \\
\hline 2 & 0.014 & - & - \\
3 & 0.018 & 0.000 & 0.018 \\
4 & 0.024 & -0.006 & 0.030 \\
\hline \hline
\end{tabular}

We have minimized the classical spin Hamiltonian (Eq.(1)) with first-principles exchange parameters in respect to the angle between different spins in the chain. On this basis we have defined the values of canting angles and net magnetic moments of the Mn nanochains. These results are presented in Table VI and Fig. 17. One can see that the net magnetic moment increases with length of the nanochain.

In order to test the reliability of the weak ferromagnetism results we have performed the $\mathrm{LDA}+\mathrm{U}+\mathrm{SO}$ calculations. For dimer system the magnetic ground state is non-collinear and spins are along $\mathrm{z}$ axis with canting of $1.0^{\circ}$ for $\mathrm{LMTO}$ and $1.6^{\circ}$ for PAW. These results are in good agreement with previous GGA calculations ${ }^{\underline{6}}$ where the easy axis of a system with single Mn atom on the $\mathrm{CuN}$ surface is $\mathrm{z}$ axis. The obtained values of canting angles are about one order larger than those obtained in our

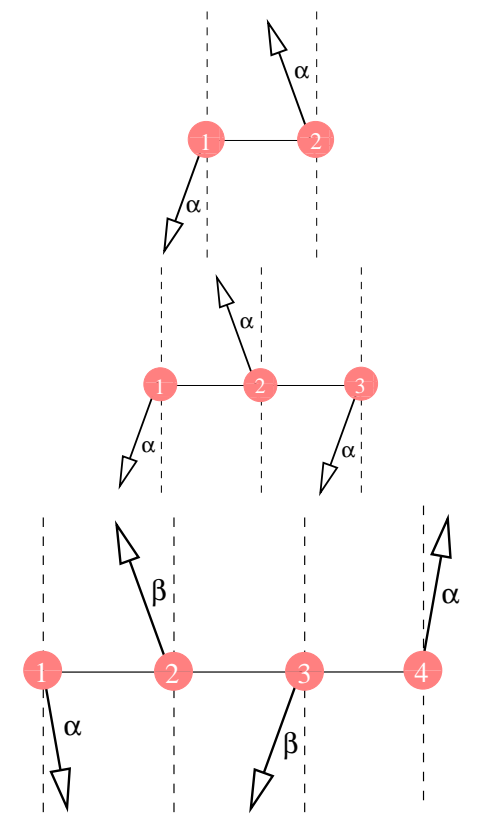

FIG. 7: Schematic representation of spin canting of dimer, trimer and quatromer systems.

LDA $+\mathrm{U}$ calculations (TableVI). This observation can be addressed to underestimation of Dzyaloshinskii-Moriya interaction calculated by Green functions method $\stackrel{21}{=}$ Obtained canting angles correspond to the following Dzyaloshinskii-Moriya interactions $D_{i j}^{x}=J_{i j} t g(\pi-2 \alpha)$ $=0.24 \mathrm{meV}$ (in LMTO) and $D_{i j}^{x}=0.34 \mathrm{meV}$ (in PAW). One can consider these results as manifestation of strong Dzyaloshinskii-Moriya interaction in investigated nanosystems.

TABLE VI: Values of canting angles and full weak ferromagnetic moments of different $\mathrm{Mn}$ nanochains (in $\mu_{B}$ ) obtained using minimization of model spin Hamiltonian.

\begin{tabular}{lcc}
\hline \hline$n$ & angle & $M_{\text {model }}$ \\
\hline 2 & $\alpha=0.057^{\circ}$ & 0.009 \\
3 & $\alpha=0.164^{\circ}$ & 0.038 \\
$4 \alpha=0.173^{\circ} \beta=0.466^{\circ}$ & 0.045 \\
\hline \hline
\end{tabular}

Clearly, the ultimate test of our results will to compare them with experiment. In the next section we will show that some experimentally observed features ${ }^{4}$ can be explained using the anisotropic exchange interaction.

\section{ZERO-FIELD SPLITTING}

In order to explain experimentally observed different zero-field energy of $m= \pm 1$ and $m=0$ excited states 4 we use the quantum spin Hamiltonian with DzyaloshinskiiMoriya interaction:

$$
\hat{H}=J \hat{\vec{S}}_{1} \hat{\vec{S}}_{2}+\vec{D}_{12}\left[\hat{\vec{S}}_{1} \times \hat{\vec{S}}_{2}\right] .
$$


For simplicity, let us consider the case of $\mathrm{S}=\frac{1}{2}$ and $\vec{D}_{12}=\left(D_{12}^{x} ; 0 ; 0\right)$. One can rewrite Eq.(11) in the following form:

$$
\hat{H}=J\left(\hat{S}_{1}^{x} \hat{S}_{2}^{x}+\hat{S}_{1}^{y} \hat{S}_{2}^{y}+\hat{S}_{1}^{z} \hat{S}_{2}^{z}\right)+D_{12}^{x}\left(\hat{S}_{1}^{y} \hat{S}_{2}^{z}-\hat{S}_{1}^{z} \hat{S}_{2}^{y}\right)
$$

The basis functions for this Hamiltonian can be written as follows,

$$
|\uparrow, \uparrow>\quad| \downarrow, \downarrow>\quad|\uparrow, \downarrow>\quad| \downarrow, \uparrow>.
$$

Using well known rules for spin operators

$$
\begin{aligned}
\hat{S}^{x}\left|\uparrow>=\frac{1}{2}\right| \downarrow> & \hat{S}^{y}\left|\uparrow>=\frac{i}{2}\right| \downarrow> & \hat{S}^{z}\left|\uparrow>=\frac{1}{2}\right| \uparrow> \\
\hat{S}^{x}\left|\downarrow>=\frac{1}{2}\right| \uparrow> & \hat{S}^{y}\left|\downarrow>=-\frac{i}{2}\right| \uparrow> & \hat{S}^{z}\left|\downarrow>=-\frac{1}{2}\right| \downarrow>
\end{aligned}
$$

one can define the matrix elements of this Hamiltonian presented in Table VII The eigenvalues of this matrix

TABLE VII: Matrix elements of the Heisenberg Hamiltonian Eq.(12)

\begin{tabular}{l|cccc}
\hline \hline & $\uparrow, \uparrow>$ & $\downarrow, \downarrow\rangle$ & $|\uparrow, \downarrow\rangle$ & $\downarrow, \uparrow>$ \\
\hline $\mid \uparrow, \uparrow>$ & $\frac{J}{4}$ & 0 & $\frac{i D_{12}^{x}}{4}$ & $-\frac{i D_{12}^{x}}{4}$ \\
$\mid \downarrow, \downarrow>$ & 0 & $\frac{J}{4}$ & $-\frac{i D_{12}^{x}}{4}$ & $\frac{i D_{12}^{x}}{4}$ \\
$\mid \uparrow, \downarrow>$ & $-\frac{i D_{12}^{x}}{4}$ & $\frac{i D_{12}^{x}}{4}$ & $-\frac{J}{4}$ & $\frac{J}{2}$ \\
$\mid \downarrow, \uparrow>$ & $\frac{i D_{12}^{x}}{4}$ & $-\frac{i D_{12}^{x}}{4}$ & $\frac{J}{2}$ & $-\frac{J}{4}$ \\
\hline \hline
\end{tabular}

are the following:

$$
\begin{array}{r}
E_{T}^{ \pm}=\frac{J}{4}, \\
E_{T}^{0}=-\frac{J}{4}+\frac{\sqrt{J^{2}+4 D^{2}}}{2}, \\
E_{S}=-\frac{J}{4}-\frac{\sqrt{J^{2}+4 D^{2}}}{2} .
\end{array}
$$

One can see that the energies of $m=0$ and $m= \pm 1$ triplet states are different. Therefore, one can expect that anisotropic exchange interaction helps us explain similar difference in the experimental spectra for the Mn dimer on the $\mathrm{CuN}$ surface.

Since in the case of $\mathrm{S}=5 / 2$ the situation is more complicated, we have numerically calculated the excitation spectra using previously obtained isotropic and anisotropic exchange interactions by means of ALPS library: 24,25 The final results are presented in Table VIII According to experiment the zero-field energies of excited states are $5.96 \pm 0.05 \mathrm{meV}$ and $5.83 \pm 0.05 \mathrm{meV}$ for $m=0$ and $m= \pm 1$, respectively, and correspond to $\Delta E=0.13 \pm 0.05 \mathrm{meV}$. One can see that the theoretically estimated values of $\Delta E$ (Table VIII) give the correct order of magnitude for experimental zero-field energy splitting. We plan to investigate the effect of single-ion magnetic anisotropy energy on this splitting.

TABLE VIII: The energy difference, $\Delta E$ of $m=0$ and $m=$ \pm 1 excited states for different sets of calculated isotropic and anisotropic exchange interactions (in $\mathrm{meV}$ ).

\begin{tabular}{cc}
\hline set & $\Delta E$ \\
\hline LMTO (LDA+U): $J=7.0, D^{x}=0.02$ & $<10^{-4}$ \\
LMTO (LDA+U+SO): $J=7.0, D^{x}=0.24$ & 0.02 \\
PAW (LDA+U+SO): $J=6.0, D^{x}=0.34$ & 0.04 \\
\hline \hline
\end{tabular}

\section{CONCLUSION}

We have performed first-principle investigations of electronic and magnetic structures of the Mn nanochains supported on the $\mathrm{CuN}$ surface. Relaxation effects have taken into consideration. The calculated isotropic exchange integrals are in good agreement with experimental data. We have also calculated the anisotropic exchange interactions in the system and predicted the antiferromagnetic ground state with weak ferromagnetism. We stress that the main source of this phenomenon is local distortion which breaks the inversion symmetry between Mn atoms. It follows that the relaxation effects are important for the system under consideration. The calculated values of canting angles are larger than those for classical antiferromagnetics with weak ferromagnetism, $\mathrm{Fe}_{2} \mathrm{O}_{3}$ and $\mathrm{La}_{2} \mathrm{CuO}_{4}$. Using calculated anisotropic exchange interactions we have explained the experimentally observed different zero-field energies of $m=0$ and $m= \pm 1$ states.

Based on our results one can expect the weak ferromagnetism phenomenon in the similar surface nanosystems. For instance, we found this spin-orbit coupling effect in Co nanochain on the Pt surface. Such work is in progress.

\section{ACKNOWLEGMENT}

We would like to thank F. Mila, M. Troyer, M. Sigrist, I.V. Solovyev and F. Lechermann for helpful discussions. The hospitality of the Institute of Theoretical Physics of Hamburg University is gratefully acknowledged. This work is supported by DFG Grant No. SFB 668-A3 (Germany), INTAS Young Scientist Fellowship Program Ref. Nr. 04-83-3230, Russian Foundation for Basic Research grant RFFI 07-02-00041, RFFI 06-02-81017, the grant program of President of Russian Federation Nr. MK1041.2007.2 and Intel Scholarship Grant. The calculations were performed on the computer cluster of "University Center of Parallel Computing" of USTU-UPI and Gonzales cluster of ETH-Zurich. 
1 Y. Zhang, N. Kohler, M. Zhang, Biomaterials 23, 1553 (2002).

2 T. Neuberger, B. Schopf, H. Hofmann, M. Hofmann, B. von Rechenberg, J. Magn. Magn. Mater. 293, 483 (2005).

3 D. Bajalan and J.A. Aziz, Progress In Electromagnetics Research Symposium (March 26-29), 461 (2006).

${ }^{4}$ C.F. Hirjibehedin, C.P.Lutz, A.J. Heinrich, Science 312, 1021 (2006).

5 C.-Y. Lin, B. Jones, A. Heinrich, APS March Meeting (2006).

${ }^{6}$ C.F. Hirjibehedin, C.-Y. Lin, A.F. Otte, M. Ternes, C.P. Lutz, B.A. Jones, A.J. Heinrich, Science 317, 1199 (2007).

7 H.J. Gotsis, N. Kioussis and D.A. Papaconstantopoulos, Phys. Rev. B 73, 014436 (2006).

8 A. Bergman, L. Nordstrom, A.B. Klautau, S. FrotaPessoaa and O. Eriksson, Phys. Rev. B 75, 224425 (2007); Phys. Rev. B 73, 174434 (2006).

9 S. Lounis, P. Mavropoulos, R. Zeller, P. H. Dederichs and S. Blügel, Phys. Rev. B 75, 174436 (2007).

10 P.E. Blöchl, Phys. Rev. B 50, 17953 (1994).

11 http://cms.mpi.univie.ac.at/vasp/

12 G. Kresse, and J. Joubert, Phys. Rev. B 59, 1758 (1999).

13 V.I. Anisimov, J. Zaanen and O.K. Andersen, Phys. Rev.
B 44, 943 (1991).

14 H.J. Monkhorst, J.D. Pack, Phys. Rev. B 135188 (1976).

15 O.K. Andersen, Phys. Rev. B 123060 (1975).

16 I. V. Solovyev, A. I. Liechtenstein, V. A. Gubanov, V. P. Antropov, O. K. Andersen, Phys. Rev. B 43, 14414 (1991).

17 A.O. Shorikov, A.V. Lukoyanov, M.A. Korotin and V.I. Anisimov, Phys. Rev. B 72, 024458 (2005).

18 W. Heisenberg, Z. Physik 49, 619 (1928).

19 Toru Moriya, Phys. Rev. 120, 91 (1960).

20 A.I. Lichtenstein, M.I. Katsnelson, V.P. Antropov, and V.A. Gubanov, J. Magn. Magn. Mater. 67, 65 (1987).

21 V.V. Mazurenko and V.I. Anisimov, Phys. Rev. B 71, 184434 (2005).

22 I.V. Solovyev, Phys. Rev. B 52, 13419 (1995).

23 I. Solovyev, N. Hamada and K. Terakura, Phys. Rev. Lett. 76, 4825 (1996)

${ }^{24}$ F. Alet et al., Phys. Rev. E 71, 036706 (2005).

${ }^{25}$ F. Alet et al., J. Phys. Soc. Jpn. Suppl. 74, 30 (2005); A. F. Albuquerque et al., J. Magn. Magn. Mater. 310, 1187 (2007); M. Troyer, B. Ammon and E. Heeb, Lecture Notes in Computer Science, 1505, 191 (1998).

26 S.-W. Cheong, M. Mostovoy, Nature 6, 13 (2007). 